\title{
Decoding Pure Rotational Molecular Spectra for Asymmetric Molecules
}

\author{
S. A. Cooke and P. Ohring \\ School of Natural and Social Sciences, Purchase College, State University of New York, 735 Anderson Hill Road, \\ Purchase, NY 10577, USA \\ Correspondence should be addressed to S. A. Cooke; stephen.cooke@purchase.edu
}

Received 24 June 2012; Accepted 30 July 2012

Academic Editor: Austin Nevin

Copyright (C) 2013 S. A. Cooke and P. Ohring. This is an open access article distributed under the Creative Commons Attribution License, which permits unrestricted use, distribution, and reproduction in any medium, provided the original work is properly cited.

Rotational spectroscopy can provide insights of unparalleled precision with respect to the wavefunctions of molecular systems that have relevance in fields as diverse as astronomy and biology. In this paper, we demonstrate how asymmetric molecular pure rotational spectra may be analyzed "pictorially" and with simple formulae. It is shown that the interpretation of such spectra relies heavily upon pattern recognition. The presentation of some common spectral line positions in near-prolate asymmetric rotational spectra provides a means by which spectral assignment, and approximate rotational constant determination, may be usefully explored. To aid in this endeavor we have created a supporting, free, web page and mobile web page.

\section{Introduction}

Microwave spectroscopy is capable of providing unique insights into the electronic structure and potential energy surfaces of molecules. It has found considerable use in the diverse fields of physics and biology, providing an invaluable method to examine the conformational landscapes of both small and large molecules. Microwave spectroscopy is a mature discipline and several recent review articles are available on the subject [1-3]. Yet recent technological advances [4], together with lower instrument costs mean that microwave spectroscopy is more accessible to researchers than it has ever been before [5-10]. The recent 2012 Microwave Newsletter [11] indicates that there are over 50 active research laboratories in locations all over the globe, with at least 400 researchers studying over 800 molecular systems. Furthermore, technology developments now allow broadband rotational spectra to be collected in a few hours, or less, meaning that undergraduate students can perform experiments in a timely fashion, that is, an undergraduate physical chemistry lab. This being so, the broadband nature of the data collected means that researchers are still faced with a complex spectrum that may not be as easily interpreted as many classroom NMR spectra or low resolution IR spectra.
The rigid rotor, as a model for a rotating linear molecule, is a subject covered in elementary quantum mechanics classes. It is unusual for classes to consider molecular asymmetry, because this is often viewed as being too complex a problem. It is the goal of this work to make this important task easier.

All types of spectroscopy require that the observed transitions, or peaks, be identified. For many types of spectroscopy spectral peak identification is associative. For example associating a specific bond stretch with a peak in an IR spectrum, or associating a methyl group with a group of peaks in an NMR spectrum. However, in many high resolution spectra, the task of transition assignment requires identifying the upper and lower quantum states involved. The spectra produced from microwave frequency studies are usually associated with the quantized rotational motions of molecules, and the task of spectral peak identification involves the latter, that is, the assignment of quantum numbers for the upper and lower states.

A rotational spectrum, like most spectra, should be viewed as a puzzle. These puzzles contain patterns, often complex in nature, that once unravelled lead to easily tractable information regarding the geometry and conformation of the subject molecule. In order for spectral patterns to be 
unravelled, the spectroscopist needs to know the "rules" of the game, that is, the underlying theory governing the asymmetric quantum rotor. For any given molecule these rules may be arrived at from an appropriately formed Hamiltonian operator, solving for the eigenvalues, and then either deriving or simply applying the appropriate selection rules. This could be called a bottom-up approach. But there is an alternative where, first, puzzle solving skills are invoked and spectral patterns identified. After this point the appropriate molecular properties may be introduced and discussed. This may be considered a top-down approach.

To this end several, powerful, software visualization tools have been developed to help with the quantum number assignment problem in pure rotational spectroscopy [12-16]. In order to compliment these tools the provision of some common spectral patterns would be useful for the beginning student.

The aim of this paper is to present some common patterns that occur in rotational spectra, together with their approximate dependence on the magnitudes of the rotational constants, so that a top down approach is more available to interested parties. Importantly, we point out that by "patterns" we are referring to transition line positions; this work does not address transition line intensities. None of the theory introduced here is new (its over 70 years old [17-19]), however, the method of presentation goes beyond that found in other sources. Through this paper it is hoped that students may be more easily introduced to rotational spectra, and that rotational spectroscopy may be more fully included in the chemistry curriculum.

\section{Background}

In order for spectral patterns to be identified, and in order to produce a self-contained paper, three concepts need to be introduced. These are (i) molecular rotational constants, (ii) the appropriate quantum numbers to use in transition assignment, and (iii) an overview of the selection rules with particular reference to how these selection rules relate to the geometry of the molecule. These are introduced in only a very cursory manner as they are treated thoroughly by several excellent monographs [20-26].

2.1. Molecular Rotational Constants. The geometry of any molecule may be reduced to three rotational constants, $A$, $B$, and $C$, which are inversely proportional to the moments of inertia about the three principal axes, $a, b$, and $c$. By convention, the $a b c$-axis system is orientated such that the $A$ rotational constant is the largest in magnitude, and $C$ is smallest. Accordingly, an important skill is to be able to locate the $a$ principal axis in such a way that it runs through, or close to, as much atomic mass as possible, followed by the $c$ principal axis such that it is perpendicular to the $a$-axis and runs through as little atomic mass as possible. For examples of this axis system Figure 1 displays three different molecules in their principal axes. The three molecules are thioxoacetaldehyde [27] (top), hexafluoroisobutene [28] (center), and 1,1,1,3,3,3hexafluoropropane [29] (bottom).
The relationship between the magnitudes of the rotational constants, that is, the shape of the molecule, governs the gross appearance of a molecule's rotational spectra. Ray's asymmetry parameter $[18], \kappa=(2 B-A-C) /(A-C)$, provides a quantitative measure of how far a molecule is from any symmetry relations. In the symmetric prolate limit where $A>B=C$, then $\kappa=-1$, whereas in the symmetric oblate limit where $A=B>C, \kappa=+1$. Figure 2 displays the moment of inertia ellipsoids, given by $x^{2} / a+y^{2} / b+z^{2} / c=1 ;\{a, b, c\} \in$ $\{A, B, C\}$, for the prolate, perfect asymmetric, and oblate tops [30]. These ellipsoids can be instructive because, whereas the rotation of a diatomic molecule is easily visualized, the rotation of an asymmetric molecule is less easily imagined. The moment of inertia ellipsoids provides a visual cue, helping students visualize asymmetric molecular rotation by imagining how the "ellipsoid rolls without slipping on a plane" [31]. Furthermore, one can see how the relationships between the moments of inertia affect the rotation.

Naturally, most molecules are asymmetric and, as may be observed in Figure 2, possess moment of inertia relationships that lie somewhere between the oblate and prolate limits. For many molecules $\kappa$ is close to -1 and they are then described as near-prolate asymmetric tops. This is a very common molecular shape and this paper focuses mainly on this type of rotational spectrum.

2.2. Rotational Quantum Numbers for Asymmetric Molecules. The rotational quantum numbers for asymmetric tops are denoted as $J_{K_{-1} K_{1}}$. The rotational quantum number $J$ is familiar from the linear rigid rotor; however, the $K_{-1}$ and $K_{1}$ labels (not quantum numbers) require brief explanation. Consider a symmetric top, either oblate or prolate. A second quantum number, $K$, is required in the energy expressions for these molecules. This quantum number pertains to the quantized rotation about the molecule's symmetry axis. The quantum number $K$ can take values of $J$ through to $-J$ in integer steps. However, only a $K^{2}$ term appears in the energy expression and so, excepting the $K=0$ levels, all $K$ levels are doubly degenerate. Now, for a prolate top, the energy levels for a specific quantum state, $J_{K}$, increase in energy from $J_{0}$ up to $J_{J}$. In stark contrast to this the oblate top energy levels, $J_{K}$, increase in energy from $J_{J}$ up to $J_{0}$.

For the asymmetric top there is no symmetry axis. This means $K$ is not a good quantum number for an asymmetric molecule. However, each energy level for the asymmetric top may be linked to (i) a $K$ value in the prolate limit, this becomes the $K_{-1}$ label and also (ii) a $K$ value in the oblate limit, which becomes the $K_{1}$ label. Essentially, we may imagine that if an asymmetric top in a $J_{K_{-1} K_{1}}$ state were to "morph" into a prolate top, then the quantum state would be $J_{K_{-1}}$. If, on the other hand, the asymmetric $J_{K_{-1} K_{1}}$ state were to "morph" into an oblate top the quantum state would be $J_{K_{1}}$.

2.3. Selection Rules. The gross selection rule for rotational spectroscopy is that the molecule must have a dipole moment. In the case of an asymmetric molecule one must consider the components of the dipole along either the $a$ and/or $b$ 


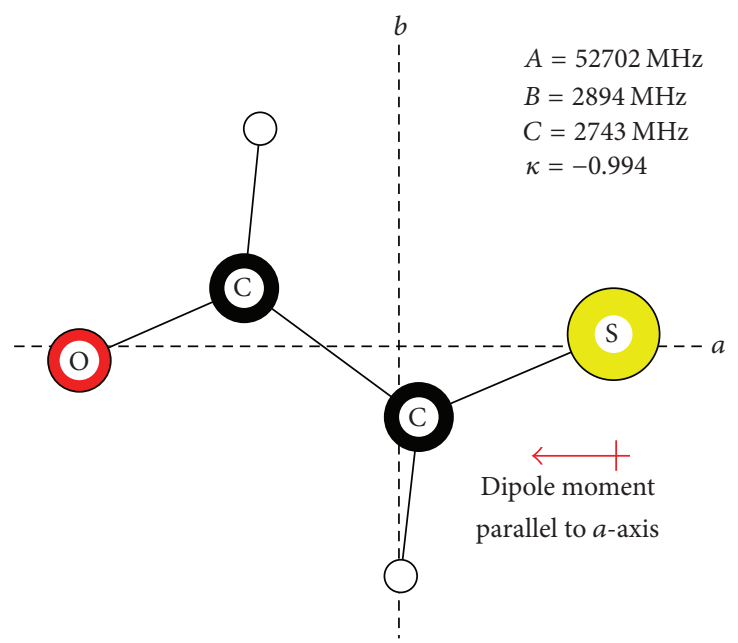

(a)

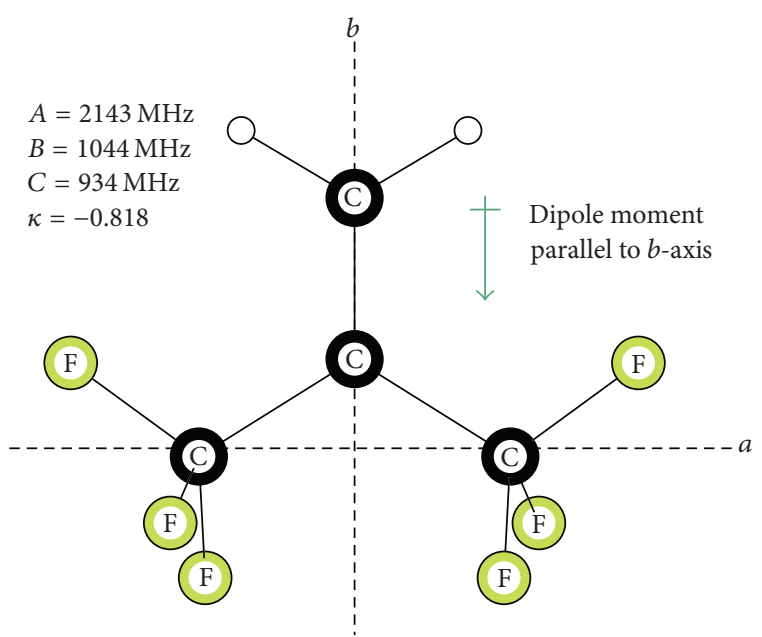

(b)

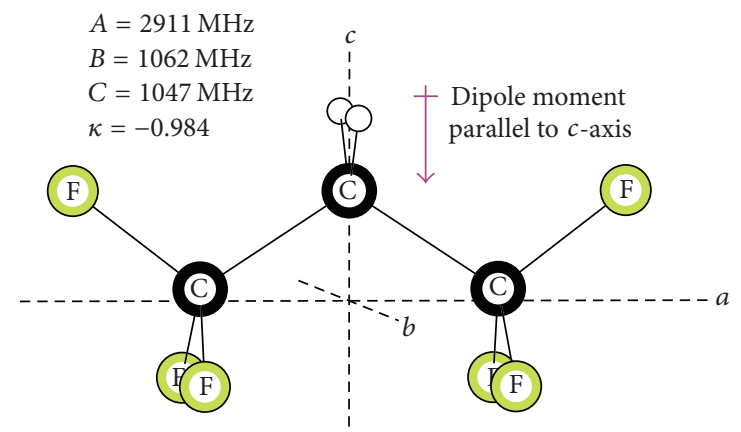

(c)

Figure 1: An example of three molecules in the $a b c$ principal axes system. The axis system is centered on the molecules center of mass. Notice how, in each case, the $a$-axis is located such that it is as close to as much atomic mass as possible. As evidenced by the inset $\kappa$ values and rotational constants, all three molecules may be considered near-prolate asymmetric rotors. The directions of the dipole components for each molecule are shown. The lines between atoms indicate connectivity and not bond order. The three molecules are thioxoacetaldehyde (a), hexafluoroisobutene (b), and 1,1,1,3,3,3-hexafluoropropane (c).

and/or $c$ principal axes. A key skill requires that electronegative/electropositive elements, or groups, be identified for the subject molecule within the $a b c$ axis system. In this way one can tell $a$ priori along which axis or axes a dipole component will be nonzero. Figure 1 shows three examples of molecules with either $a$-, $b$-, or $c$-dipole components.

A molecular rotation about the $a$ principal axis places different symmetry requirements on the relation between upper and lower wavefunctions in a rotational transition compared to a rotation about either the $b$ or $c$ axes. These requirements are manifest in the relation between the upper and lower quantum states $K_{-1} K_{1}$ labels. It is the parity of the labels, that is, even $(e)$ or odd $(o)$, that we concern ourselves with. In short, for a molecular rotation about the $a$-axis, along which there is a dipole component, a change in parity is only allowed for the $K_{1}$ label. This is referred to as an $a$-type transition. For a $c$-type transition, a change in parity is only allowed in the $K_{-1}$ label. For a $b$-type transition, a change in parity must occur in the $K_{-1}$ and $K_{1}$ labels simultaneously.

In regards to the changes permitted in the rotational quantum number $J$ it may be shown that $\Delta J=-1,0,+1$.
Here $\Delta J$ means $J$ (upper state, higher energy) $-J$ (lower state, lower energy). Just as is the case for vibration-rotation spectroscopy, transitions of the type $\Delta J=-1$ are referred to as $P$-branch transitions, and by extension $\Delta J=0$ is the $Q$ branch, and $\Delta J=+1$ is the $R$-branch. In passing we note that, in our experience, $Q$ - and $R$-branch transitions are by far the most commonly observed in pure rotational spectra.

Lastly, a shorthand notation for any transition, or group of transitions, is commonly used. By example, an ${ }^{a} R_{0,1}$ transition is an $a$-type, $R$-branch transition in which the value of $K_{-1}$ does not change between the lower and upper states, and the $K_{1}$ label increases by 1 between lower and upper states.

\section{A Note on Centrifugal Distortion}

In this work we have assumed the rotating molecule to be rigid. Real molecules do, however, undergo distortions in their average nuclear positions as rotation occurs. This is referred to as centrifugal distortion and is accounted for in a descriptive set of molecular parameters by several 


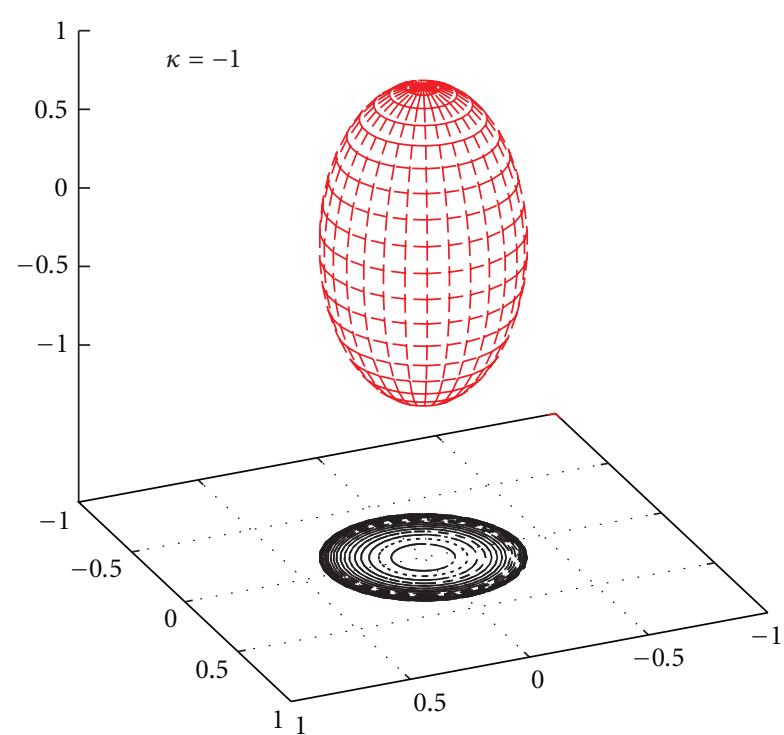

(a)

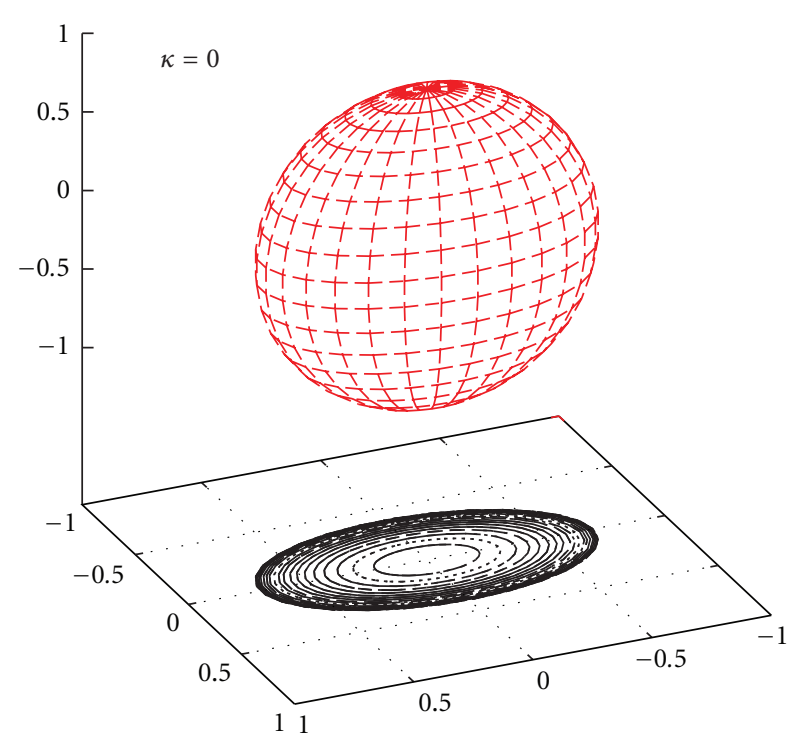

(b)

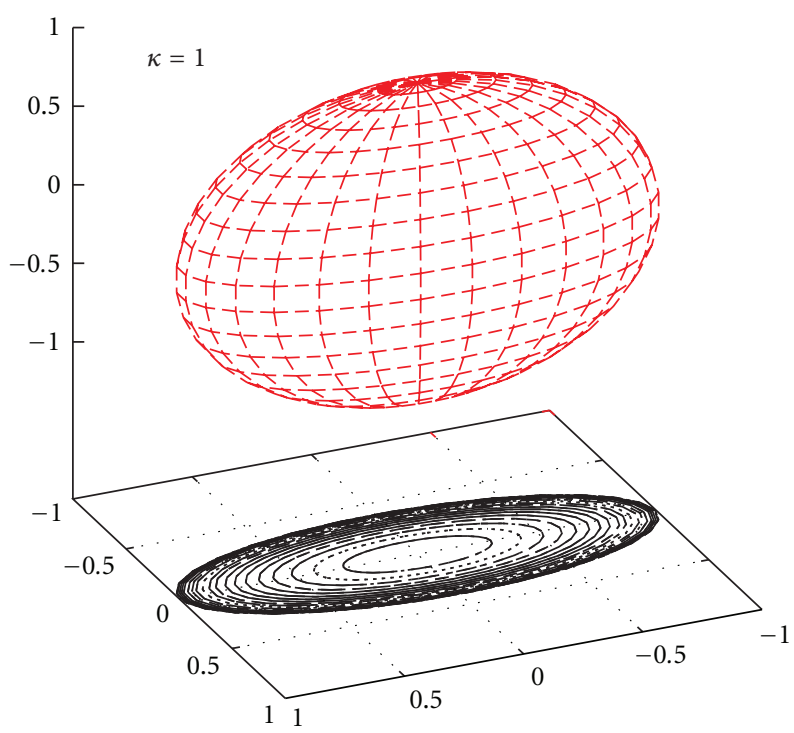

(c)

FIGURE 2: The moment of inertia ellipsoids for symmetric prolate (a), asymmetric (b), and symmetric oblate (c) molecular rotors.

centrifugal distortion constants [32]. The magnitudes of these constants are, crudely, inversely proportional to the square of the molecular mass. The centrifugal distortion constants are many orders of magnitude smaller than the rotational constants. The effect of centrifugal distortion on the observed spectra is to change the frequency of a given rigid rotational transition. These effects are generally minimal, that is, a few $\mathrm{kHz}$ or so for low $J$ transitions, but can become significant, that is, many $\mathrm{MHz}$, for high $J$ transitions. They have been ignored in this work because at low $J$, that is, $J$ less than, say 10 , centrifugal distortion does not affect the patterns of rotational transitions observed. However, with modern microwave spectrometers, the precision of measurement of rotational transitions is such that the effects of centrifugal distortion are routinely observed and must be accounted for in the Hamiltonian operator.

\section{Rotational Spectra}

In the following we have selected some common transition series observed in rotational spectra for asymmetric molecules. The transitions are discussed using the notation $J_{K_{-1} K_{1}}^{\prime} \leftarrow J_{K_{-1} K_{1}}^{\prime \prime}$, where the double prime indicates the lower state.

We describe them pictorially and by the provision of simple formulae. It should once again be noted that effects of centrifugal distortion have been neglected. The spectra 
TABle 1: Summary of transition patterns and features.

\begin{tabular}{|c|c|c|}
\hline Transition type & Frequency & Notes \\
\hline \multicolumn{3}{|c|}{$R$-branch, $J^{\prime}=J^{\prime \prime}+1$} \\
\hline$a$-type & $(B+C)\left(J^{\prime \prime}+1\right)$ & $\begin{array}{c}\text { For each, } J^{\prime} \leftarrow J^{\prime \prime} \text {, with } J^{\prime \prime}>0, \\
\text { transitions are spread over }(B-C)\left(J^{\prime \prime}+1\right)\end{array}$ \\
\hline $\begin{array}{l}b \text {-type } \\
\quad \begin{array}{l}\prime \\
J_{1, J^{\prime}}^{\prime \prime}\end{array} \\
J_{0, J^{\prime}}^{\prime} \leftarrow J_{0, J^{\prime \prime}}^{\prime \prime}\end{array}$ & $\begin{array}{c}A+C+2 C J^{\prime \prime} \\
(3 B+2 C-A)+2 B\left(J^{\prime \prime}-1\right)\end{array}$ & \\
\hline $\begin{array}{l}J_{2, J^{\prime}-1}^{\prime} \leftarrow J_{1, J^{\prime \prime}-1}^{\prime \prime} \\
J_{2, J^{\prime}-2}^{\prime} \leftarrow J_{1, J^{\prime \prime}}^{\prime \prime}\end{array}$ & $(3 A+C+(B-C) / 2)+(B+C)\left(J^{\prime \prime}-1\right)$ & Doublet split by $((B-C) / 2) J^{\prime} J^{\prime \prime}$ \\
\hline $\begin{array}{l}J_{3, J^{\prime}-2}^{\prime} \leftarrow J_{2, J^{\prime \prime}-2}^{\prime \prime} \\
J_{3, J^{\prime}-3}^{\prime} \leftarrow J_{2, J^{\prime \prime}-1}^{\prime \prime}\end{array}$ & $(5 A+C+(B-C) / 2)+(B+C)\left(J^{\prime \prime}-2\right)$ & Very closely spaced doublets \\
\hline 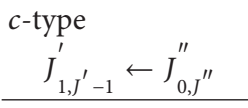 & $A+B+2 B J^{\prime \prime}$ & \\
\hline \multicolumn{3}{|c|}{$\frac{A+B+2 B J}{Q-\text { branch, } J^{\prime}=J^{\prime \prime}}$} \\
\hline \multicolumn{3}{|l|}{ a-type } \\
\hline$J_{1, J-1} \leftarrow J_{1, J}$ & $J=2: v=3(B-C)$ & $J=2: v(J)=v(J-1)+(B-C) J$ \\
\hline$J_{J, 1} \leftarrow J_{J-2,2}$ & $2(A-2(B+C))(J-1)$ & Groups of same $K_{-1}$ \\
\hline \multicolumn{3}{|l|}{$b$-type } \\
\hline$J_{2, J-2} \leftarrow J_{1, J-1}$ & $J=2: v=3(A-B)$ & $J>2: v(J)=v(J-1)-((B-C) / 2) J$ \\
\hline$J_{2, J-1} \leftarrow J_{1, J}$ & $J=2: v=3(A-C)$ & $J>2: v(J)=v(J-1)+((B-C) / 2) J$ \\
\hline
\end{tabular}

displayed in Figures 3 through 8 were calculated exactly using the rotational constants provided in each figure using a complete Hamiltonian matrix diagonalization routine [33, 34]. The calculated spectra relate to molecules at rotational temperatures of $3 \mathrm{~K}$, which is a common temperature for operating a modern microwave spectrometer, where molecules are entrained in supersonic expansions of an inert gas. The simple formulae provided have been determined through consideration of both the closed algebraic expressions for the lowest $J$ energy levels [21] and also through $a$ posteriori examination of exact spectra. A useful compilation of empirical relations for the frequencies of rotational spectral transitions is provided in Table 7.11 in "Microwave Molecular Spectra" by Gordy and Cook [26]. However, to the best of our knowledge, many of the formulae provided here appear in print for the first time.

In regards to strategy, assignment should begin by examining the entire rotational spectrum and looking for repeating patterns. If patterns can be located they may be compared to the portions of spectra displayed in Figures 3 through 8 . Once patterns have been identified the empirical relations provided below, and summarized in Table 1, allow initial approximations of the rotational constants $A, B$, and $C$. With initial estimates of the rotational constants further patterns may be searched for and the process repeated in a bootstrap fashion.

\subsection{R-Branch Transitions}

4.1.1. a-Types. These transitions are perhaps the easiest to locate in a broadband spectrum. The ease with which they may be located is based on their regular harmonic pattern. They occur in groups centered at approximately $(B+C)\left(J^{\prime \prime}+\right.$ 1 ), that is, every $B+C \mathrm{MHz}$ a similar looking group is observable. Each group spans $(B-C)\left(J^{\prime \prime}+1\right)$. A sample of one of these groups is shown in Figure 3 for three different asymmetries. It is observed that the strongest transition in this group, the $J_{0, J^{\prime}}^{\prime} \leftarrow J_{0, J^{\prime \prime}}^{\prime \prime}$ transition, moves to lower frequency, away from the center of the group, as $\kappa$ moves further away from -1 .

Within each group of transitions, and when the $A$ rotational constant is of modest magnitude compared to $B$ and $C$, transitions involving high values of the $K_{-1}$ label are observable as doublets in a characteristic pattern. The pattern contains unresolved doublets, involving the highest $K_{-1}$ values, emerging from approximately $(B+C)\left(J^{\prime \prime}+1\right)$. The pattern progresses to higher frequencies, with doublets appearing with increased splittings as $K_{-1}$ decreases in value. An example is shown in Figure 4, and the pattern is also visible in Figure 3. For low $J$ transitions, that is, $J^{\prime \prime}<5$, the splitting in the $K_{-1}=2$ doublet is crudely given by 


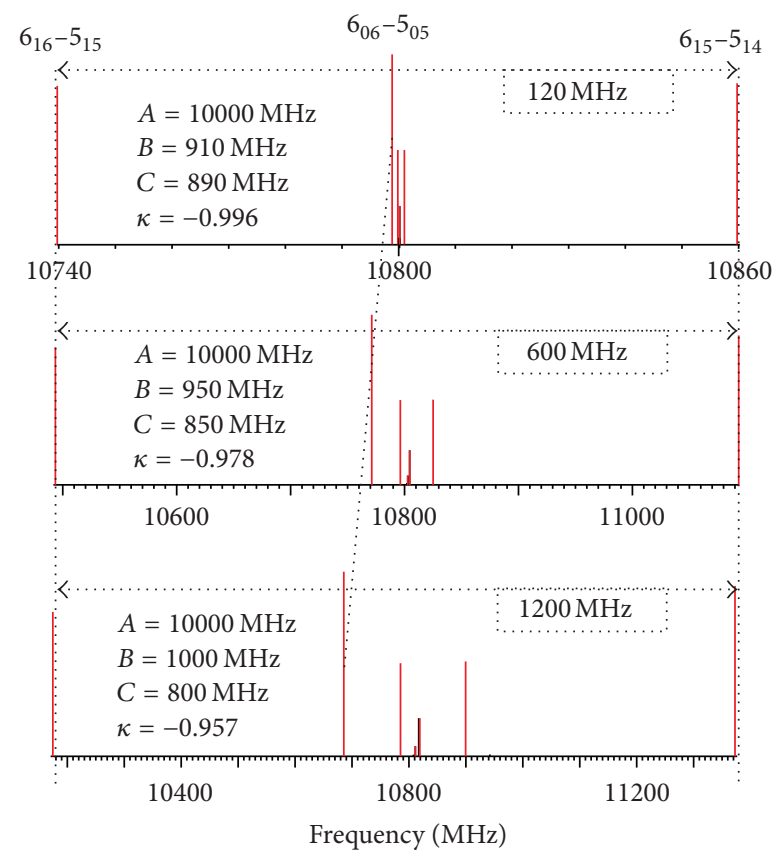

Figure 3: An example of a common pattern for $a$-type $R$-branch transitions. The three patterns show the effects of increasing asymmetry as the figure is viewed from top to bottom. The center of the group is located at $(B+C)\left(J^{\prime \prime}+1\right)$, the spread of each group is equal to $(B-C)\left(J^{\prime \prime}+1\right)$. The values of rotational constants used to generate the spectrum are shown.

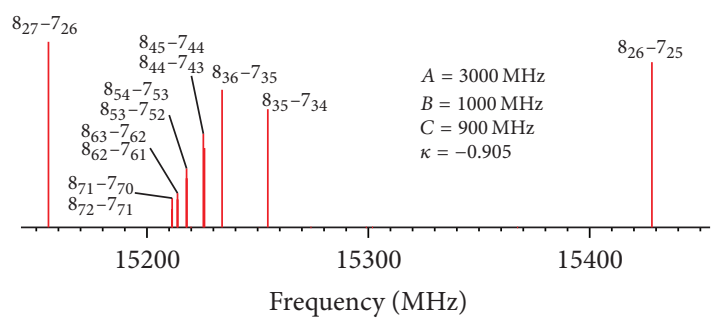

FIgURE 4: A second example of a common pattern for $a$-type $R$ branch transitions in which the $K_{-1}$ label takes values larger than 2. The transitions appear as doublets starting with the highest $K_{-1}$ doublet located at approximately $(B+C)\left(J^{\prime \prime}+1\right)$ with the successive lower $K_{-1}$ doublets occurring at higher frequency and increased splitting.

$\left[(B-C)^{2} / A\right]\left(J^{\prime \prime}+1\right)$. For higher $J$ transitions this estimate is approximately one order of magnitude too small.

\subsection{2. b-Types}

(1) $J_{1, J^{\prime}}^{\prime} \leftarrow J_{0, J^{\prime \prime}}^{\prime \prime}$. The lowest frequency member of this group of transitions, that is, the $1_{11} \leftarrow 0_{00}$ transition, occurs at $A+C$. Transitions in this family then progress to higher frequencies

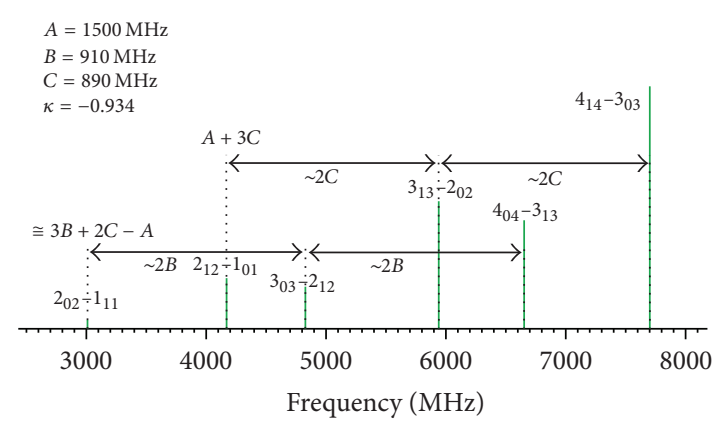

FIGURE 5: Common $b$-type $R$-branch transition patterns. Two sequences are shown, firstly the $J_{1, J^{\prime}} \leftarrow J_{0, J^{\prime \prime}}^{\prime \prime}$ transitions with approximate general formula $A+C+2 C J^{\prime \prime}$, and secondly the $J_{0, J^{\prime}}^{\prime} \leftarrow$ $J_{1, J^{\prime \prime}}^{\prime \prime}$ transitions with approximate general formula $3 B+2 C-A+$ $2 B\left(J^{\prime \prime}-1\right)$.

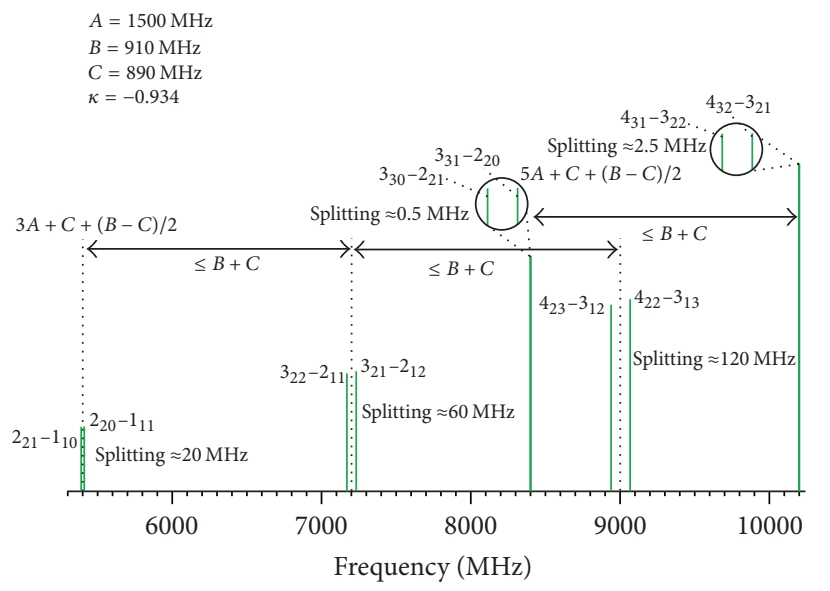

FIgURE 6: Higher $K_{-1} b$-type $R$-branch transition patterns. Two sequences are shown pertaining to $K^{\prime}{ }_{-1}=2$ and 3 , the text should be consulted for further description. These doublets are often useful in making quantum number assignments.

spaced by $2 C$, giving a general formula of $A+C+2 C J^{\prime \prime}$. An example of the progression is shown in Figure 5.

(2) $J_{0, J^{\prime}}^{\prime} \leftarrow J_{1, J^{\prime \prime}}^{\prime \prime}$. The lowest frequency member of this group of transitions, that is, the $2_{02} \leftarrow 1_{11}$ transition, occurs at $3 B+2 C-A$. Transitions in this family progress to higher frequencies spaced by $2 B$. This results in a general formula of $3 B+2 C-A+2 B\left(J^{\prime \prime}-1\right)$. An example of the progression is shown in Figure 5.

(3) $J_{2, J^{\prime}-1}^{\prime} \leftarrow J_{1, J^{\prime \prime}-1}^{\prime \prime}$ and $J_{2, J^{\prime}-2}^{\prime} \leftarrow J_{1, J^{\prime \prime}}^{\prime \prime}$. For a given $J^{\prime \prime}$ these two transition types appear as characteristic doublets within the rotational spectrum. The lowest pair of transitions allowed are the $2_{21} \leftarrow 1_{10}$ and the $2_{20} \leftarrow 1_{11}$ transitions. The center frequency of the doublets in this family of transitions is given by $(3 A+C+(B-C) / 2)+(B+C)\left(J^{\prime \prime}-1\right)$. The splitting between the doublets is given by approximately $((B-C) / 2) J^{\prime} J^{\prime \prime}$, that is, half of $B-C$, multiplied by the 


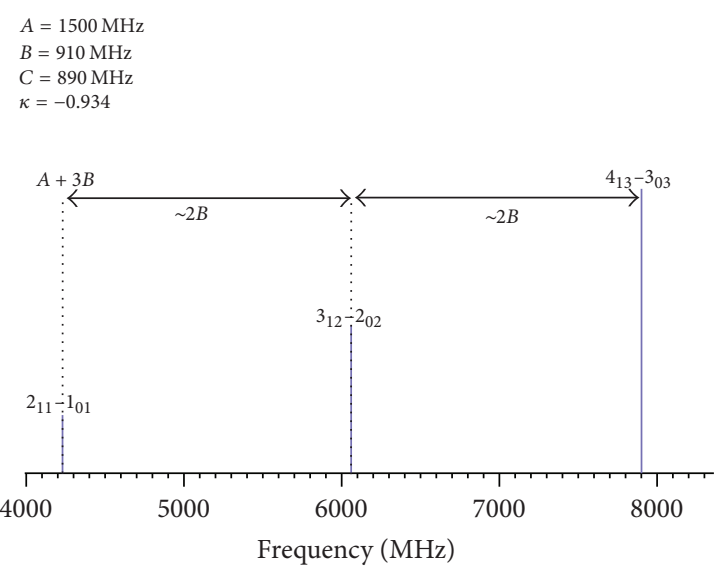

Figure 7: A common $c$-type $R$-branch transition pattern. The $J_{1, J^{\prime}-1}^{\prime} \leftarrow J_{0, J^{\prime \prime}}^{\prime \prime}$ transitions have an approximate general formula of $A+B+2 B J^{\prime \prime}$.

product of the upper and lower $J$ values. Given the dependence of the frequency on $3 \mathrm{~A}$, these transitions often fall at higher frequencies than the previously listed transitions. For example, for the $\mathrm{CH}_{3} \mathrm{CHO}$ the $2_{21} \leftarrow 1_{10}$ transition falls at approximately $179 \mathrm{GHz}$. An example of the progression is shown in Figure 6.

(4) $J_{3, J^{\prime}-2}^{\prime} \leftarrow J_{2, J^{\prime \prime}-2}^{\prime \prime}$ and $J_{3, J^{\prime}-3}^{\prime} \leftarrow J_{2, J^{\prime \prime}-1}^{\prime \prime}$. For a given $J^{\prime \prime}$ these two transitions appear as very closely spaced doublets. For values of $\kappa$ approaching -1 , where $B-C$ is small, the doublets are often not resolvable. The center frequency for the doublets appearing in this family is given by $(5 A+C+$ $(B-C) / 2)+(B+C)\left(J^{\prime \prime}-1\right)$. As was the case for the previous set of transitions, these doublets often fall at high frequencies, owing to their dependence on $5 \mathrm{~A}$. An example of the progression is shown in Figure 6.

4.1.3. c-Types. (1) $J_{1, J^{\prime}-1}^{\prime} \leftarrow J_{0, J^{\prime \prime}}^{\prime \prime}$. The lowest frequency member of this group of transitions, that is, the $1_{10} \leftarrow 0_{00}$ transition, occurs at $A+B$. Transitions in this family then progress to higher frequencies spaced by $2 B$, giving a general formula of $A+B+2 B J^{\prime \prime}$. An example of the progression is shown in Figure 7. We note in passing that it is not uncommon for $c$-type transitions and $b$-type transitions of the type listed above to occur in similar patterns. Caution should be made to ensure that the nonzero dipole components of the molecule under study are identified prior to analysis (see Figure 1).

\subsection{Q-Branch Transitions}

\subsection{1. a-Types}

(1) $J_{1, J-1} \leftarrow J_{1, J}$. The lowest frequency of these transitions, with $J=2$, occurs at $3(B-C)$. For $J>2$ the transition frequencies increase according to the recursion formula $v(J)=v(J-1)+(B-C) J$. For near prolate cases $B-C$ is

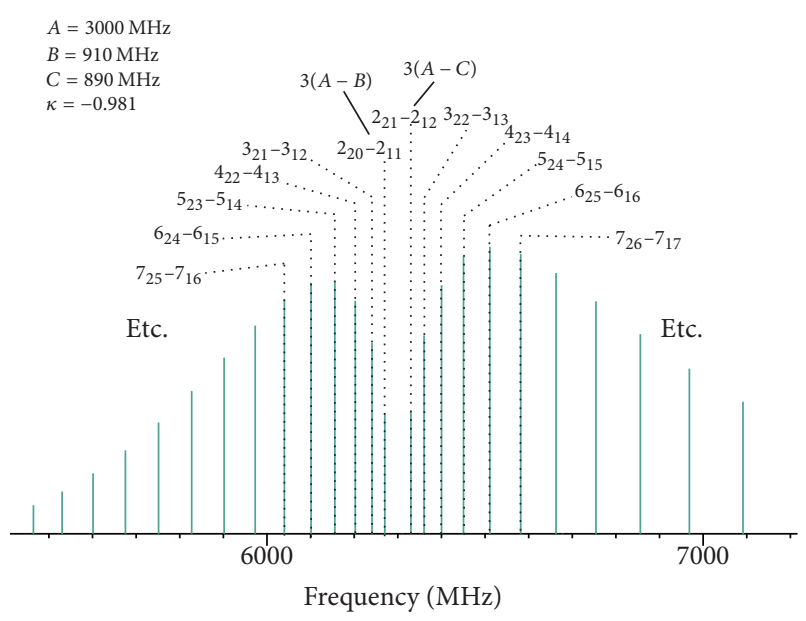

Figure 8: An example of a $b$-type $Q$-branch progression of transitions.

a small number and accordingly only very high $J$ transitions would fall in observable regions where they will likely be low in intensity.

(2) $J_{J, 1} \leftarrow J_{J-2,2}$. For low $J$ transitions the formula (4A-(B+ $C) / 2)(J-1)$ works reasonably well. Transitions are found as closely spaced groups where the $J$ level changes, the $K_{-1}$ does not change, and the $K_{1}$ level increases by 1 . For example, the $3_{31} \leftarrow 3_{12}$ transition will be found close by (a few tens of $\mathrm{MHz}$ ) to the $4_{32} \leftarrow 4_{13}$ transition and so on.

4.2.2. b-Types. These transitions produce very distinct patterns in rotational spectra. An example is shown in Figure 8. Transitions of the type $J_{2, J-2} \leftarrow J_{1, J-1}$ "appear," that is, when $J=2$, at frequency of $3(A-B)$. The transitions then "fan" out to lower frequencies with increasing $J$ according to the recursion formula $v(J)=v(J-1)-((B-C) / 2) J$. For molecules possessing small $A$ rotational constants, that is, $A<3 \mathrm{GHz}$, the next group of transitions, for which $K_{-1}=3$, occur at approximately $A-(B-C) / 2$ higher in frequency.

Related transitions of the type $J_{2, J-1} \leftarrow J_{1, J}$ "appear," that is, when $J=2$, at at frequency of $3(A-C)$. These transitions fan out to higher frequencies with increasing $J$ according to the recursion formula $v(J)=v(J-1)+((B-C) / 2) J$. Again, for molecules possessing small $A$ rotational constants, that is, $A<3 \mathrm{GHz}$, the next group of transitions, for which $K_{-1}=3$, occur at approximately $A-(B-C) / 2$ higher in frequency.

For the higher $K_{-1}$ members of these types of transitions, the pattern observed is such that increasingly higher $J$ transitions appear at increasingly lower frequencies as doublets, whereby the two transition types mentioned immediately above, begin to overlap one another.

4.2.3. c-Types. In many cases the $c$-type Q-branch transitions appear at frequencies very similar to those outlined previously for $b$-type Q-branch transitions. However, in our experience, molecules for which $c$-type Q-branch transitions 
are important in the initial stages of quantum number assignments are very rare.

\section{How Well, and under What Restrictions, Do These Simple Formulae Work?}

The line positions, described by the formulae above, are summarized in Table 1 and shown in Figures 3 to 8 . To test the performance of these relations the following procedure was performed. A simple FORTRAN 77 program was written (an Excel spreadsheet, or MathCad program, would achieve the same purpose) to use the relationships in Table 1 to generate the transition frequencies for a total of 75 transitions of $J$ up to 10 , at different values of Ray's asymmetry parameter, $\kappa$ from -1 to -0.8 . The frequencies generated were compared to the exact frequencies for the transitions generated by a complete Hamiltonian matrix diagonalization routine using Herb Pickett's SPCAT program $[33,34]$. For each $\kappa$ value, and for the three cases $J^{\prime}<10$ (75 transitions), $J^{\prime}<6$ (43 transitions), and $J^{\prime}<4$ (23 transitions), a root-meanstandard deviation was calculated according to

$$
\mathrm{RMSD}=\sqrt{\frac{\left[\sum(v(\text { act })-v(\text { est }))^{2}\right]}{N}},
$$

where $N$ is the number of transitions. The results are shown in Figure 9. It is not at all surprising that the simple formulae provided work poorly at high $J$ and high asymmetries. If one sets a quantitative limit of desiring the predicted transitions to fall, on average, within $50 \mathrm{MHz}$ of their actual location, then it is found that it is only possible to work with high $J$ transitions, that is, $J^{\prime}$ up to 9 , when $\kappa$ is between -0.975 and $\approx-1$. If, on the other hand, it is necessary to work with an asymmetric species where $\kappa$ is -0.8 , or even lower in magnitude, then it is found that the formulae presented produce transition frequencies within $50 \mathrm{MHz}$ of the actual frequencies only for transitions involving $J^{\prime}$ less than or equal to 3 .

This may appear very unsatisfactory. However, the situation is better than it seems when one recalls, the very thesis of this paper, that it is the pattern of transitions that is more informative in regards to quantum number assignments than the absolute frequencies. Figure 10 shows this comparison. The upper portion of Figure 10 shows a rotational spectrum calculated exactly using SPCAT. The lower portion shows a stick spectrum produced from the relationships given in Table 1 and the same rotational constants. The match is sufficient that a quantum number assignment could likely be made for over $50 \%$ of the transitions shown.

\section{New Media Support}

For quantum number assignment problems, students are encouraged to plot stick spectra using the formulae given in Table 1 . However, in order to rapidly visualize the patterns of transitions formed by the relationships given in Table 1 both a web-based visualization tool [35] and matching smartphone mobile web page [36] are being developed. Using either

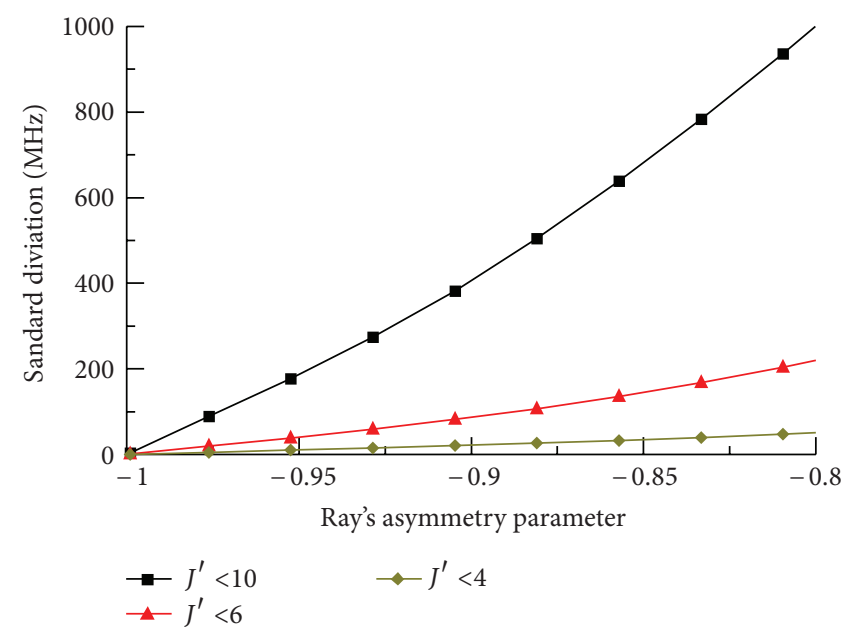

FIgURE 9: A plot of the root-mean-standard deviation in $\mathrm{MHz}$ pertaining to the comparison of actual rigid rotor frequencies compared to those generated using the simple formulae given in Table 1 for several different molecular asymmetries.

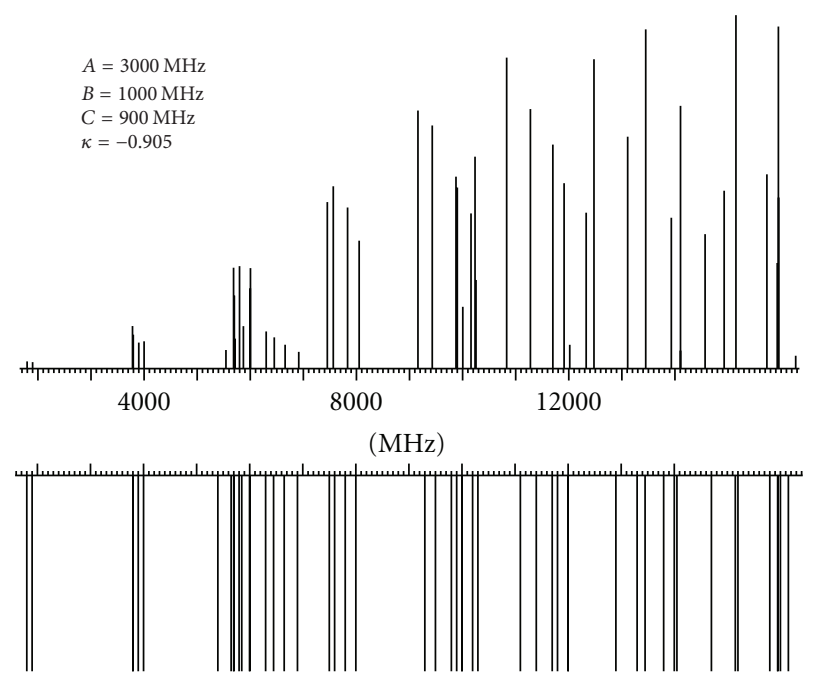

FIGURE 10: The actual rigid rotor spectrum arising from the rotational constants given (top), compared to the complete rigid rotor spectrum generated using the same rotational constants and the simple formulae given in Table 1 (bottom).

medium, users can enter a set of rotational constants, press "Plot Spectrum," and then see the patterns of low $J$ transitions that result from the relationships given in Table 1. Only low $J$ transitions are shown in order to maintain a low spectral density. The transitions displayed are colorcoded so that the $a$-type transitions are shown in red, the $b$-type transitions are in green, and the $c$-type transitions are in yellow. A screenshot of the smartphone application is provided in Figure 11. The display format follows closely that of the excellent AABS software package for the assignment of broadband microwave spectra developed by Professor Kisiel [12, 13]. Users are encouraged to contact the authors with comments regarding improvements. 


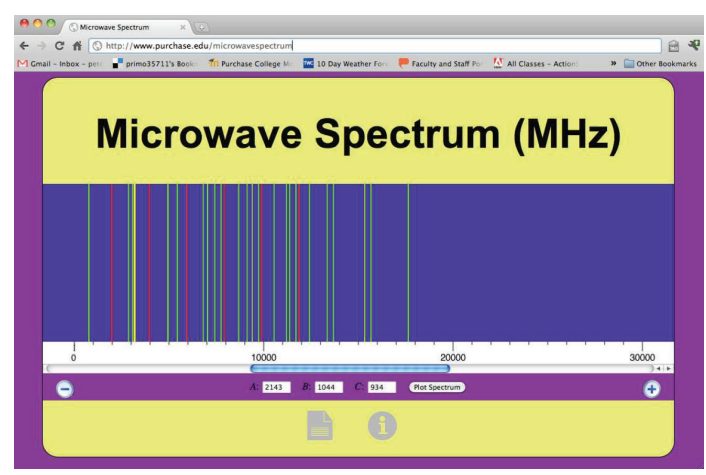

FIGURE 11: Screenshot of the smartphone mobile website supporting this work. Users may enter values for the three rotational constants in $\mathrm{MHz}$ and then plot the patterns of transitions that result from the formulae of Table 1.

\section{Conclusions}

Rotational spectra may be inspected, initially, so as to locate repeating patterns of transitions. We have presented "template" spectra indicating the types of patterns that can commonly occur for the near-prolate asymmetric molecule. Location of these patterns in experimental spectra can help leading to succesful quantum number assignments and/or initial evaluations of the subject molecule's rotational constants.

The information provided allows students to adopt an alternative approach to considering the quantum mechanical, asymmetric rigid rotor. In this method students are actively involved early, that is, in the task of spectral assignment. Web and smartphone resources have been created to assist in this endeavor. The tools provided facilitate, and prompt, in depth discussion about the under lying theory and application of microwave spectroscopy.

\section{Acknowledgments}

We are grateful to Mr. Frank DeChirico, Mr. Bryan Harrison, and Professor S. E. Novick for proof-reading this paper and their improving comments.

\section{References}

[1] N. R. Walker, Philosophical Transactions of the Royal Society A, vol. 365, no. 1861, pp. 2813-2828, 2007.

[2] J.-U. Grabow and W. Caminati, "Microwave spectroscopy: experimental techniques," in Frontiers of Molecular Spectroscopy, J. Laane, Ed., p. 383, 2009.

[3] J.-U. Grabow and W. Caminati, "Microwave spectroscopy: molecular systems," in Frontiers of Molecular Spectroscopy, J. Laane, Ed., p. 455, 2009.

[4] G. G. Brown, B. C. Dian, K. O. Douglas, S. M. Geyer, S. T. Shipman, and B. H. Pate, "A broadband Fourier transform microwave spectrometer based on chirped pulse excitation," Review of Scientific Instruments, vol. 79, Article ID 053103, 13 pages, 2008.
[5] M. D. Marshall, H. O. Leung, B. Q. Scheetz, J. E. Thaler, and J. S. Muenter, "A chirped pulse Fourier transform microwave study of the refrigerant alternative 2,3,3,3-tetrafluoropropene," Journal of Molecular Spectroscopy, vol. 266, no. 1, pp. 37-42, 2011.

[6] L. Evangelisti, G. Sedo, and J. van Wijngaarden, "Rotational spectrum of 1,1,1-trifluoro-2-butanone using chirped-pulse Fourier transform microwave spectroscopy," Journal of Physical Chemistry A, vol. 115, no. 5, pp. 685-690, 2011.

[7] D. A. Obenchain, A. A. Elliott, A. L. Steber et al., "Rotational spectrum of three conformers of 3,3-difluoropentane: construction of a $480 \mathrm{MHz}$ bandwidth chirped-pulse Fourier-transform microwave spectrometer," Journal of Molecular Spectroscopy, vol. 261, no. 1, pp. 35-40, 2010.

[8] B. Reinhold, I. A. Finneran, and S. T. Shipman, "Room temperature chirped-pulse Fourier transform microwave spectroscopy of anisole," Journal of Molecular Spectroscopy, vol. 270, no. 2, pp. 89-97, 2011.

[9] A. L. McJunkins and G. G. Brown, "An inexpensive roomtemperature chirped-pulse fourier transform microwave (RTCP-FTMW) spectrometer," Journal of Undergraduate Chemistry Research, vol. 10, p. 174, 2011.

[10] G. S. Grubbs II, C. T. Dewberry, K. C. Etchison, K. E. Kerr, and S. A. Cooke, "A search accelerated correct intensity Fourier transform microwave spectrometer with pulsed laser ablation source," Review of Scientific Instruments, vol. 78, no. 9, Article ID 096106, 3 pages, 2007.

[11] R. K. Bohn, Micowave Spectroscopy Information Letter LV, 2012.

[12] Z. Kisiel, L. Pszczólkowski, I. R. Medvedev, M. Winnewisser, F. C. D. Lucia, and E. Herbst, "Rotational spectrum of trans-trans diethyl ether in the ground and three excited vibrational states," Journal of Molecular Spectroscopy, vol. 233, no. 2, pp. 231-243, 2005.

[13] Z. Kisiel, "PROSPE-Programs for ROtational SPEctroscopy," http://www.ifpan.edu.pl/ kisiel/prospe.htm.

[14] C. M. Western, "PGOPHER, a Program for Simulating Rotational Structure," University of Bristol, 2011, http://pgopher .chm.bris.ac.uk.

[15] I. R. Medvedev, M. Winnewisser, B. P. Winnewisser, F. C. D. Lucia, and E. Herbst, "The use of CAAARS (Computer Aided Assignment of Asymmetric Rotor Spectra) in the analysis of rotational spectra," Journal of Molecular Structure, vol. 742, no. 1-3, pp. 229-236, 2005.

[16] D. F. Plusquellic, "JB95, Spectral Fitting Program," 2009, http:// www.nist.gov/pml/div682/grp01/jb95.cfm.

[17] S. C. Wang, "On the asymmetrical top in quantum mechanics," Physical Review, vol. 34, no. 2, pp. 243-252, 1929.

[18] B. S. Ray, "Über die Eigenwerte des asymmetrischen Kreisels," Zeitschrift für Physik, vol. 78, no. 1-2, pp. 74-91, 1932.

[19] G. W. King, R. M. Hainer, and P. C. Cross, "I. Calculation and symmetry classification of energy levels," The Journal of Chemical Physics, vol. 11, no. 1, pp. 27-42, 1943.

[20] M. W. P. Strandberg, Microwave Spectroscopy, John Wiley \& Sons, New York, NY, USA, 1954.

[21] C. H. Townes and A. L. Schawlow, Microwave Spectroscopy, Dover Publications, New York, NY, USA, 1955.

[22] H. C. Allen Jr. and P. C. Cross, Molecular Vib-Rotors, John Wiley \& Sons, New York, NY, USA, 1963.

[23] J. E. Wollrab, Rotational Spectra and Molecular Structure, Academic Press, New York, NY, USA, 1967. 
[24] W. H. Flygare, Molecular Structure and Dynamics, Prentice Hall, New Jersey, NJ, USA, 1978.

[25] H. W. Kroto, Molecular Rotation Spectra, Wiley, New York, NY, USA, 1975.

[26] W. Gordy and R. L. Cook, Microwave Molecular Spectra: Techniques of Chemistry Vol. XVIII, Wiley, New York, NY, USA, 1984.

[27] D. McNaughton and D. N. Bruget, "The microwave spectrum of thioglyoxal (thioxo-acetaldehyde) (SCHCHO)," Journal of Molecular Spectroscopy, vol. 134, no. 1, pp. 129-133, 1989.

[28] S. A. Cooke, S. E. Novick, and W. C. Pringle, Unpublished results.

[29] M. Onda, K. Tsuda, and E. Sakamoto, "The microwave spectrum and structure of 1,1,1,3,3,3-hexafluoropropane, freon R236fa," Journal of Molecular Structure, vol. 780-781, pp. 222-224, 2006.

[30] A. I. Borisenko, I. E. Tarapov, and R. A. Silverman, Vector and Tensor Analysis with Applications, Dover Publishers, New York, NY, USA, 1979.

[31] W. Gordy, W. V. Smith, and R. F. Trambarulo, Microwave Spectroscopy, Dover Publication, New York, NY, USA, 1966.

[32] J. K. G. Watson, Vibrational Spectra and Structure Vol. 6, Elsevier, Amsterdam, The Netherlands, 1978, Edited by: J. R. Durig.

[33] H. M. Pickett, "The fitting and prediction of vibration-rotation spectra with spin interactions," Journal of Molecular Spectroscopy, vol. 148, no. 2, pp. 371-377, 1991.

[34] H. M. Pickett, “SPFIT/SPCAT package," http://spec.jpl.nasa gov.

[35] P. Ohring, 2012, http:/ffaculty.purchase.edu/peter.ohring/microwavespectrum.

[36] P. Ohring, 2012, http://faculty.purchase.edu/peter.ohring/microwavespectrummobile. 

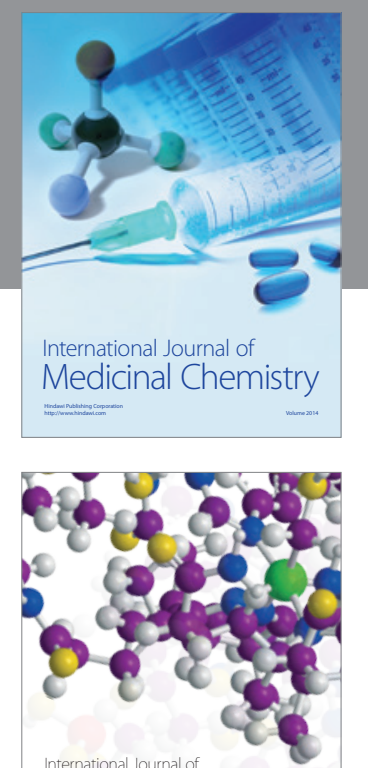

\section{Carbohydrate} Chemistry

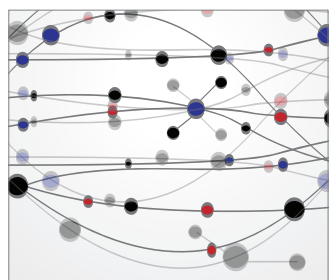

The Scientific World Journal
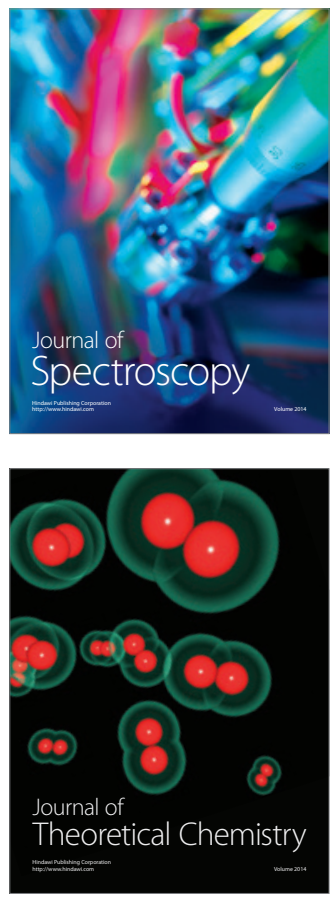
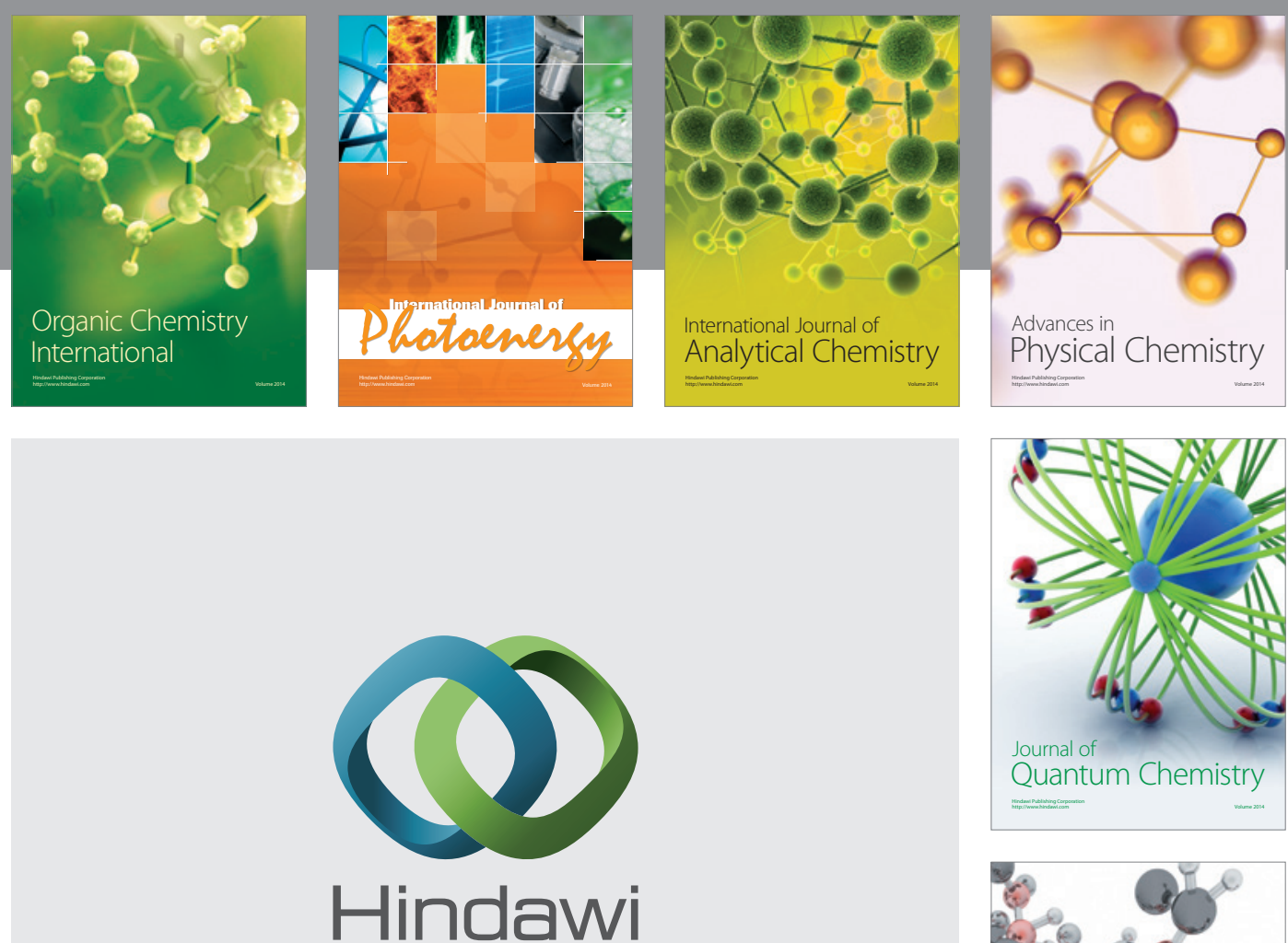

Submit your manuscripts at

http://www.hindawi.com

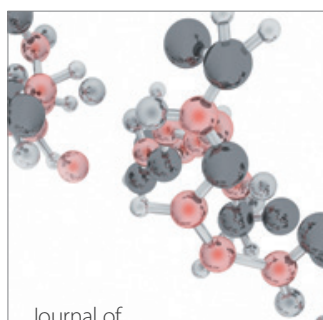

Analytical Methods

in Chemistry

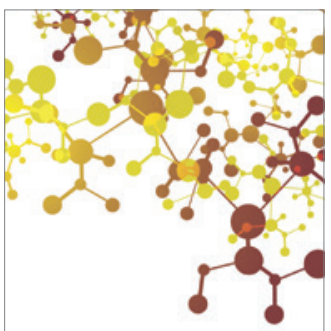

Journal of

Applied Chemistry

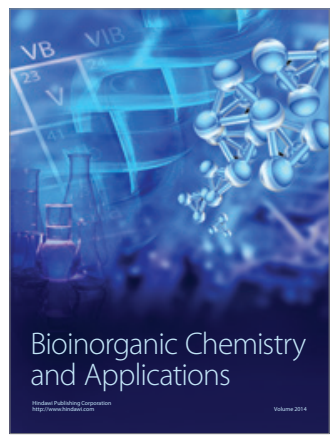

Inorganic Chemistry
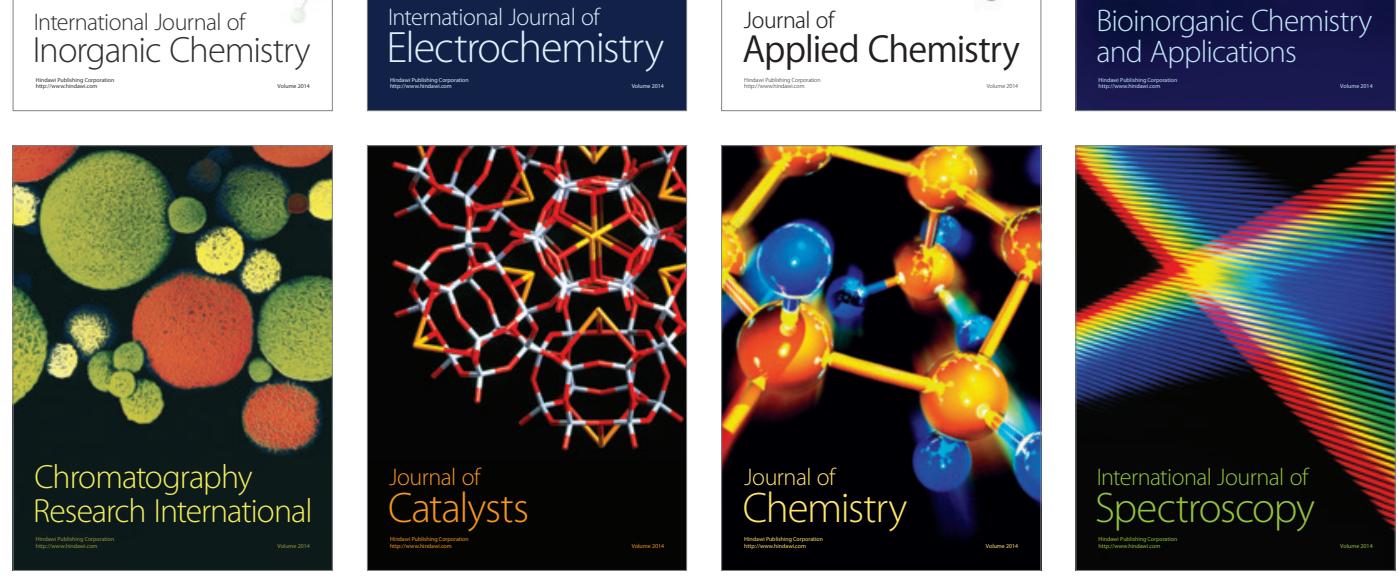